\title{
ANALYTIC $L$-FUNCTIONS: DEFINITIONS, THEOREMS, AND CONNECTIONS
}

\author{
DAVID W. FARMER, AMEYA PITALE, NATHAN C. RYAN, AND RALF SCHMIDT
}

\begin{abstract}
L$-functions can be viewed axiomatically, such as in the formulation due to Selberg, or they can be seen as arising from cuspidal automorphic representations of GL $(n)$, as first described by Langlands. Conjecturally, these two descriptions of $L$-functions are the same, but it is not even clear that these are describing the same set of objects. We propose a collection of axioms that bridges the gap between the very general analytic axioms due to Selberg and the very particular and representation-theoretic construction due to Langlands. Along the way we prove theorems about $L$-functions that satisfy our axioms and state conjectures that arise naturally from our axioms.
\end{abstract}

\section{Contents}

1. Introduction

2. Two views of $L$-functions

3. $\mathbb{Q}$-automorphic $L$-functions and the axioms

4. Algebraic and arithmetic $L$-functions

5. Connections

Appendix A. Nontempered $L$-functions $\quad 277$

About the authors

References

\section{INTRODUCTION}

Different mathematicians mean different things when they say " $L$-function". Some mean an element of the Selberg class and others might mean a Dirichlet series with Euler product and require that it be associated to an automorphic form. For some people an $L$-function has to be entire, for others it can have poles on the edge of the critical strip, for yet others it can even have poles in other locations.

In this paper we show how one can attach adjectives to $L$-functions (and which adjectives one should attach, as determined by one's goals) in such a way that the resulting classes of $L$-functions provide a detailed framework for understanding $L$ functions. This framework can be used to clarify the distinctions between various classes and also to unify by showing connections between them. In Section 2, we define the following sets of $L$-functions (see Section 2 for definitions):

Received by the editors December 9, 2017.

2010 Mathematics Subject Classification. Primary 11M06, 11M41, 11F66, 11F03, 11F70. 


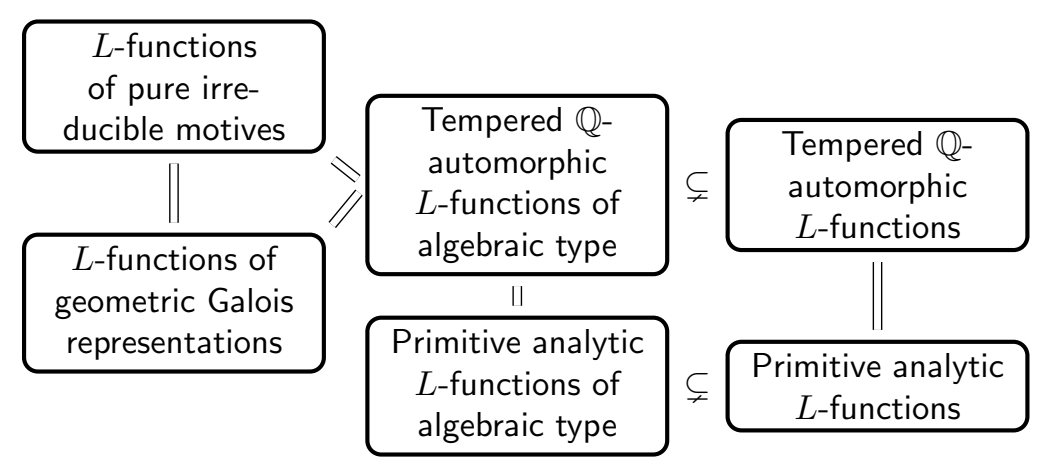

Figure 1. Conjectured relationships between the sets of $L$ functions considered in this paper.

- Tempered balanced analytic primitive entire L-functions. These L-functions are defined axiomatically, with precise restrictions on their functional equation and Euler product.

- $\mathbb{Q}$-automorphic L-functions. These $L$-functions are associated to tempered balanced unitary cuspidal automorphic representations of $\mathrm{GL}\left(n, \mathbb{A}_{\mathbb{Q}}\right)$.

The above sets are believed to contain all primitive $L$-functions that are expected to satisfy analogues of the Riemann hypothesis, and conjecturally the two sets are essentially equal.

Within each of the above sets are distinguished subsets which, conjecturally, contain all $L$-functions arising from arithmetic objects.

- L-functions of algebraic type (analytic and $\mathbb{Q}$-automorphic). These are characterized by conditions on the $\Gamma$-factors in the functional equation.

- L-functions of arithmetic type (analytic and $\mathbb{Q}$-automorphic). These are characterized by conditions on the coefficients in the Dirichlet series.

Conjecturally, all four of these sets of $L$-functions are equal and arise from the following arithmetic objects:

- pure motives;

- geometric Galois representations.

Associated to each such arithmetic object is an $L$-function. Conjecturally, those sets of $L$-functions are equal, and coincide with the four subsets of $L$-functions mentioned previously. The conjectured relationships between these sets of $L$-functions is shown in Figure 1

In Figure 2 in Section 5 we discuss in more detail the conjectured relationships between the sets of $L$-functions described above. Precise descriptions of each of these sets are given in Sections 2 and 4.

\section{TWO VIEWS OF $L$-FUnCTIONS}

2.1. Analytic $L$-functions. The first set of $L$-functions in our discussion is defined axiomatically.

Throughout the axioms, $s=\sigma+i t$ is a complex variable with $\sigma$ and $t$ real.

A tempered balanced analytic $L$-function is a function $L(s)$ which satisfies the five axioms below. In this paper we will also refer to tempered analytic L-functions and analytic L-functions, which are obtained by relaxing some of these axioms; see Section 2.1.1 for a discussion. 
Axiom 1 (Analytic properties). $L(s)$ is given by a Dirichlet series

$$
L(s)=\sum_{n=1}^{\infty} \frac{a_{n}}{n^{s}}
$$

where $a_{n} \in \mathbb{C}$.

(a) Convergence: $L(s)$ converges absolutely for $\sigma>1$.

(b) Analytic continuation: $L(s)$ continues to a meromorphic function having only finitely many poles, with all poles lying on the $\sigma=1$ line.

Axiom 2 (Functional equation). There is a positive integer $N$ called the conductor of the L-function, a positive integer $d$ called the degree of the $L$ function, a pair of nonnegative integers $\left(d_{1}, d_{2}\right)$ called the signature of the $L$-function (where $d=d_{1}+2 d_{2}$ ), and complex numbers $\left\{\mu_{j}\right\}_{j=1}^{d_{1}}$ and $\left\{\nu_{k}\right\}_{k=1}^{d_{2}}$ called the spectral parameters of the L-function, such that the completed $L$-function

$$
\Lambda(s)=N^{s / 2} \prod_{j=1}^{d_{1}} \Gamma_{\mathbb{R}}\left(s+\mu_{j}\right) \prod_{k=1}^{d_{2}} \Gamma_{\mathbb{C}}\left(s+\nu_{k}\right) \cdot L(s)
$$

has the following properties:

(a) Bounded in vertical strips: Away from the poles of the L-function, $\Lambda(s)$ is bounded in vertical strips $\sigma_{1} \leq \sigma \leq \sigma_{2}$.

(b) Functional equation: There exists $\varepsilon \in \mathbb{C}$, called the sign of the functional equation, such that

$(\mathrm{Ax} 2.2)$

$$
\Lambda(s)=\varepsilon \bar{\Lambda}(1-s) .
$$

Axiom 3 (Euler product). There is a product formula

$$
L(s)=\prod_{p \text { prime }} F_{p}\left(p^{-s}\right)^{-1},
$$

absolutely convergent for $\sigma>1$.

(a) Polynomial: $F_{p}$ is a polynomial with $F_{p}(0)=1$.

(b) Degree: Let $d_{p}$ be the degree of $F_{p}$. If $p \nmid N$, then $d_{p}=d$, and if $p \mid N$, then $d_{p}<d$.

Axiom 4 (Temperedness). The spectral parameters and Satake parameters satisfy precise bounds:

(a) Selberg bound: For every $j$ we have $\operatorname{Re}\left(\mu_{j}\right) \in\{0,1\}$ and $\operatorname{Re}\left(\nu_{k}\right) \in$ $\left\{\frac{1}{2}, 1, \frac{3}{2}, 2, \ldots\right\}$.

(b) Ramanujan bound: Write $F_{p}$ in factored form as

$$
F_{p}(z)=\left(1-\alpha_{1, p} z\right) \cdots\left(1-\alpha_{d_{p}, p} z\right)
$$

with $\alpha_{j, p} \neq 0$. If $p \nmid N$, then $\left|\alpha_{j, p}\right|=1$ for all $j$. If $p \mid N$, then $\left|\alpha_{j, p}\right|=p^{-m_{j} / 2}$ for some $m_{j} \in\{0,1,2, \ldots\}$, and $\sum m_{j} \leq d-d_{p}$.

Axiom 5 (Central character). There exists a Dirichlet character $\chi \bmod N$, called the central character of the L-function.

(a) Highest degree term: For every prime $p$,

$$
F_{p}(z)=1-a_{p} z+\cdots+(-1)^{d} \chi(p) z^{d} .
$$

(b) Balanced: We have $\operatorname{Im}\left(\sum \mu_{j}+\sum\left(2 \nu_{k}+1\right)\right)=0$.

(c) Parity: The spectral parameters determine the parity of the central character

$$
\chi(-1)=(-1)^{\sum \mu_{j}+\sum\left(2 \nu_{k}+1\right)} .
$$


2.1.1. Comments on the terminology. The term balanced is described by Axiom 5 (b). The summand " +1 " can obviously be omitted from the condition, but we include it for uniformity with Axiom 5(c). Note that Axiom 5(c) would be problematic if it were not assumed that the exponent on -1 was an integer. If we omit the modifier "balanced" when describing an $L$-function, then we mean a function of the form $L(s+i y)$, where $L(s)$ is balanced and $y \in \mathbb{R}$. If $L(s)$ is a (not necessarily balanced) $L$-function, then it is straightforward to check that there exists exactly one $y_{0} \in \mathbb{R}$ such that $L\left(s+i y_{0}\right)$ is balanced.

The term tempered refers to both the Selberg bound in Axiom 4(a) and the Ramanujan bound in Axiom 4(b). Neither bound has been proven for most automorphic $L$-functions, but if those axioms fail for an automorphic $L$-function, they must fail in a specific way arising from the fact that the underlying representation is unitary. A precise description of the possibilities is given by the unitary pairing condition, described in Appendix $\mathrm{A}$.

In the functional equation $\mathrm{Ax2.2}$, the function $\bar{\Lambda}$ is the Schwartz reflection of $\Lambda$, defined for arbitrary analytic functions $f$ by $\bar{f}(z)=\overline{f(\bar{z})}$. The tuple

$$
\left(\varepsilon, N,\left\{\mu_{1}, \ldots, \mu_{d_{1}}\right\},\left\{\nu_{k}, \ldots, \nu_{d_{2}}\right\}\right)
$$

is the functional equation data of the $L$-function. The sign $\varepsilon$ of the functional equation has absolute value 1: to see this, apply the functional equation twice to get $\Lambda(s)=\varepsilon \bar{\varepsilon} \Lambda(s)$.

In the Euler product, the polynomials $F_{p}$ are known as the local factors, and the reciprocal roots $\alpha_{j, p}$ are called the Satake parameters at $p$. If $p \mid N$, then we say $p$ is a bad prime, and if $p \nmid N$, then $p$ is good.

It follows straight from the definition that if $L_{1}(s)$ and $L_{2}(s)$ are analytic $L$ functions, then so is $L_{1}(s) L_{2}(s)$. And if both $L_{1}$ and $L_{2}$ are balanced, or tempered, then so is their product. If the analytic $L$-function $L(s)$ cannot be written nontrivially as $L(s)=L_{1}(s) L_{2}(s)$, then we say that $L$ is primitive. Here "nontrivially" refers to the fact that the constant function 1 is a degree $0 L$-function.

Because the degree is additive, every $L$-function can be written as a finite product of primitive $L$-functions. It is not obvious that the factors are unique, and indeed this has not been shown without additional assumptions, such as the following conjecture of Selberg.

Conjecture 2.1 (Selberg orthogonality). Suppose $L_{1}(s)=\sum a_{n} n^{-s}$ and $L_{2}(s)=$ $\sum b_{n} n^{-s}$ are primitive analytic (not necessarily tempered or balanced) L-functions. Then

$$
\sum_{p<X} \frac{a_{p} \overline{b_{p}}}{p}=\delta_{L_{1}, L_{2}} \log \log X+O(1),
$$

where $\delta_{L_{1}, L_{2}}=1$ if $L_{1}=L_{2}$ and 0 otherwise.

Selberg orthogonality was originally conjectured [24] for the Selberg class, which includes an assumption of temperedness. In the general (not necessarily tempered) case, one might consider including prime powers in the sum (2.1). If one has sufficient progress toward temperedness, such as $\theta<\frac{1}{4}$ (see Appendix A.2 for the definition of $\theta$ ), or an averaged version, such as Rudnick and Sarnak's "Hypothesis H" 21], then such prime power terms do not contribute to the sum. We have omitted such terms for the practical matter that the prime sum version (2.1) gives a straightforward proof of unique factorization. 
Theorem 2.2. If $L(s)$ is an analytic L-function, then

$$
L(s)=L_{1}(s) \cdots L_{n}(s),
$$

where each $L_{j}$ is a nontrivial primitive analytic $L$-function. If $L(s)$ is tempered, then each $L_{i}(s)$ is tempered. Assuming the Selberg orthogonality conjecture, the representation is unique, except for the order of the factors.

Proof. The first statement is immediate from the definition of primitivity and the fact that the degree of a product is the sum of the degrees of the factors. The second statement is immediate from the definition of tempered. Assuming Selberg orthogonality, uniqueness follows from [8, proof of Proposition 4.4].

If "balanced" is included as a condition in Theorem 2.2, then the conclusion can be written as

$$
L(s)=L_{1}\left(s+i y_{1}\right) \cdots L_{n}\left(s+i y_{n}\right),
$$

where each $L_{j}$ is balanced, $y_{j} \in \mathbb{R}$, and $\sum d_{j} y_{j}=0$, where $d_{j}$ is the degree of $L_{j}$. Examples such as $\zeta(s+5 i) \zeta(s-5 i)$ and $L(s+6 i, \chi) L(s-3 i, E)$ show that a nonprimitive balanced $L$-function cannot necessarily be written as a product of primitive balanced $L$-functions.

The motivation for the central character axioms comes from the $\mathbb{Q}$-automorphic $L$-functions that we describe in the next section; see the discussion preceding equations (3.3) and (3.5), and note, in particular, that the discussion explains why Axioms 5(a) and 5(b) are equivalent if the $L$-function is $\mathbb{Q}$-automorphic.

2.2. $\mathbb{Q}$-automorphic $L$-functions. For a number field $F$, let $\mathbb{A}_{F}$ denote the ring of adeles of $F$. In this section we consider $L$-functions of cuspidal automorphic representations $\pi$ of the group $\operatorname{GL}\left(n, \mathbb{A}_{\mathbb{Q}}\right)$. For such $\pi$ we will always use the same conventions as in [7]; in particular, we assume $\pi$ to be irreducible and unitary. Then $\pi$ admits a unitary central character $\omega_{\pi}$, which is a character of

$$
\mathbb{Q}^{\times} \backslash \mathbb{A}_{\mathbb{Q}}^{\times}=\mathbb{R}_{>0} \times \prod_{p<\infty} \mathbb{Z}_{p}^{\times}
$$

There exists a unique real number $y$ and a character $\chi$ of $\mathbb{Q}^{\times} \backslash \mathbb{A}_{\mathbb{Q}}^{\times}$of finite order such that $\omega_{\pi}=|\cdot|{ }^{i y} \chi$. The character $\chi$ corresponds to a Dirichlet character, also denoted by $\chi$ :

$$
\omega_{\pi}(x)=x^{i y} \quad \text { for } x \in \mathbb{R}_{>0} .
$$

We say $\pi$ is balanced if the restriction of $\omega_{\pi}$ to $\mathbb{R}_{>0}$ is trivial; that is, if $y=0$. Evidently, this is equivalent to $\omega_{\pi}$ being of finite order. In this case $\omega_{\pi}$ corresponds to a Dirichlet character $\chi$. The correspondence is such that if $\omega_{\pi}$ factors as $\prod_{p \leq \infty} \omega_{\pi, p}$, then

$$
\omega_{\pi, p}(p)=\chi(p)
$$

for all primes $p$ not dividing the conductor of $\chi$, and

$$
\omega_{\pi, \infty}(-1)=\chi(-1) .
$$

Definition 2.3. Let $\pi=\bigotimes_{p \leq \infty} \pi_{p}$ be a cuspidal automorphic representation of $\operatorname{GL}\left(d, \mathbb{A}_{\mathbb{Q}}\right)$. Let

$$
L(s, \pi)=\prod_{p<\infty} L\left(s, \pi_{p}\right)
$$


be the finite part of the Langlands $L$-function associated to $\pi$ with respect to the standard representation of the dual group $\mathrm{GL}(d, \mathbb{C})$. We call $L(s, \pi)$ a $\mathbb{Q}$ automorphic L-function.

We do not consider automorphic representations for groups other than GL $(n)$ or for number fields other than $\mathbb{Q}$. This is not a serious restriction since more general automorphic representations can always be transferred to $\operatorname{GL}(n)$ over $\mathbb{Q}$, at least conjecturally; see [1] for some recent and deep results. This transfer may not be cuspidal, however, so our definition will exclude some more general automorphic $L$-functions. The following examples will illustrate why restricting to $\operatorname{GL}(n)$ over $\mathbb{Q}$ is not harmful, and in fact it is desirable for our purposes.

First consider Hilbert modular cuspforms on a real quadratic number field $F$. As explained in [19], such modular forms correspond to cuspidal automorphic representations $\pi$ of $\mathrm{GL}\left(2, \mathbb{A}_{F}\right)$. The $L$-function $L(s, \pi)$ is a degree $2 L$-function over $F$. We may consider the automorphic induction $\mathcal{A} \mathcal{I}_{F / \mathbb{Q}}(\pi)$, which is an automorphic representation of $\mathrm{GL}\left(4, \mathbb{A}_{\mathbb{Q}}\right)$. It has the same $L$-function $L(s, \pi)$, but now we consider it as a degree $4 L$-function over $\mathbb{Q}$. If $\mathcal{A I}_{F / \mathbb{Q}}(\pi)$ is cuspidal, then $L(s, \pi)$ is included in our definition of automorphic $L$-function. Assume that $\mathcal{A} \mathcal{I}_{F / \mathbb{Q}}(\pi)$ is not cuspidal. Then, by [2, §3.6], $\pi$ is Galois invariant, and therefore it is in the image of the base change map from $\mathrm{GL}\left(2, \mathbb{A}_{\mathbb{Q}}\right)$ to $\mathrm{GL}\left(2, \mathbb{A}_{F}\right)$. It follows that there exists a cuspidal, automorphic representation $\pi_{\mathbb{Q}}$ of $\mathrm{GL}\left(2, \mathbb{A}_{\mathbb{Q}}\right)$ such that $L(s, \pi)=L\left(s, \pi_{\mathbb{Q}}\right) L\left(s, \pi_{\mathbb{Q}} \otimes \chi_{F / \mathbb{Q}}\right)$, where $\chi_{F / \mathbb{Q}}$ is the quadratic character corresponding to the extension $F / \mathbb{Q}$. We will later compare the class of $L$-functions according to Definition 2.3 with the class of primitive analytic $L$-functions. It is therefore advantageous to exclude a nonprimitive example such as $L(s, \pi)$ from our definition of automorphic $L$-functions.

As our second example, consider Siegel modular cuspforms $F$ of degree 2. Such $F$ correspond to cuspidal automorphic representations $\pi$ of $\operatorname{GSp}\left(4, \mathbb{A}_{\mathbb{Q}}\right)$, which, at least conjecturally, can be transferred to $\mathrm{GL}\left(4, \mathbb{A}_{\mathbb{Q}}\right)$. For example, if $F$ has full level and is not a Saito-Kurokawa lifting, then the transfer is established in [18. This transfer is again cuspidal, and thus the spin (degree 4$) L$-function $L(s, \pi)$ is included in Definition 2.3. Assume however that $F$ is a Saito-Kurokawa lifting. Then the transfer to $\mathrm{GL}\left(4, \mathbb{A}_{\mathbb{Q}}\right)$ still exists but is no longer cuspidal. Hence, in this case, $L(s, \pi)$ is not included in our definition of automorphic $L$-function. This is desirable, since $L(s, \pi)$ is of the form $\zeta\left(s-\frac{1}{2}\right) \zeta\left(s+\frac{1}{2}\right) L(s, f)$, which is neither primitive nor satisfies the Ramanujan condition. Thus, it should be excluded from our comparison with the class of primitive tempered analytic $L$-functions.

We see from these examples that $L$-functions of cuspidal automorphic representations are in general not primitive, in the sense that they may factor as products of $L$-functions of smaller degrees. The following lemma shows that this cannot happen for $\mathbb{Q}$-automorphic $L$-functions.

Lemma 2.4. Let $\pi$ be a cuspidal automorphic representation of $\mathrm{GL}\left(d, \mathbb{A}_{\mathbb{Q}}\right)$. Then the $\mathbb{Q}$-automorphic $L$-function $L(s, \pi)$ is primitive; i.e., if

$$
L(s, \pi)=\prod_{j=1}^{m} L\left(s, \pi_{j}\right)
$$

with cuspidal automorphic representations $\pi_{j}$ of $\mathrm{GL}\left(d_{j}, \mathbb{A}_{\mathbb{Q}}\right)\left(d_{j}>0\right)$ for $1 \leq j \leq m$, then $m=1$. 
Proof. We consider partial $L$-functions and twist (2.7) by the contragredient $\pi_{1}^{\vee}$ of $\pi_{1}$ :

$$
L_{S}\left(s, \pi \times \pi_{1}^{\vee}\right)=\prod_{j=1}^{m} L_{S}\left(s, \pi_{j} \times \pi_{1}^{\vee}\right) .
$$

By [7, Corollary to Theorem 2.4], $L_{S}\left(s, \pi_{1} \times \pi_{1}^{\vee}\right)$ has a pole at $s=1$. By [27, Theorem 5.2], $L_{S}\left(s, \pi_{j} \times \pi_{1}^{\vee}\right)$ has no zeros on $\operatorname{Re}(s)=1$ for any $j$. It follows that the right-hand side of (2.8) has a pole at $s=1$. Hence, so does the left-hand side, which again by [7, Theorem 2.4] implies that $\pi=\pi_{1}$. In other words, we must have $m=1$.

Remark. For an alternative proof of Lemma 2.4. see [16].

\section{Q-Automorphic $L$-FunCtions And The AXIOMS}

Now that we have defined $\mathbb{Q}$-automorphic $L$-functions and have identified a collection of axioms for analytic $L$-functions, we begin to show that $\mathbb{Q}$-automorphic $L$-functions satisfy the axioms.

Theorem 3.1. Let $L(s, \pi)$ be a $\mathbb{Q}$-automorphic $L$-function. There is a positive integer $N$, a pair of nonnegative integers $\left(d_{1}, d_{2}\right)$ so that $d_{1}+2 d_{2}=d$, and complex numbers $\left\{\mu_{j}\right\}$ and $\left\{\nu_{j}\right\}$ such that

$$
\Lambda(s, \pi)=N^{s / 2} \prod_{j=1}^{d_{1}} \Gamma_{\mathbb{R}}\left(s+\mu_{j}\right) \prod_{j=1}^{d_{2}} \Gamma_{\mathbb{C}}\left(s+\nu_{j}\right) \cdot L(s, \pi)
$$

has the following properties:

(1) $\Lambda(s, \pi)$ is entire.

(2) $\Lambda(s, \pi)$ is bounded in vertical strips $\sigma_{1} \leq \sigma \leq \sigma_{2}$.

(3) There exists $\varepsilon \in \mathbb{C}$ such that

$$
\Lambda(s, \pi)=\varepsilon \bar{\Lambda}(1-s, \pi) .
$$

(4) If $\pi$ is balanced, then $L(s, \pi)$ is balanced.

In other words, $\mathbb{Q}$-automorphic $L$-functions satisfy Axioms 1 and 2 as described in Section 2.1

Proof. For items (11), (21), and (3) above, see [7, Theorems 2.3 and 2.4]. Note that the functional equation in [7] is written as $\Lambda(s, \pi)=\varepsilon \Lambda(1-s, \tilde{\pi})$, where $\tilde{\pi}$ is the contragredient representation; one can show that $\Lambda(1-s, \tilde{\pi})=\bar{\Lambda}(1-s, \pi)$ for unitary $\pi$ (recall that $\bar{f}(s)=\overline{f(\bar{s})}$ for a function of a complex variable).

Item (4) follows by considering the local Langlands parameter at the archimedean place, keeping in mind that the determinant of this parameter corresponds to the central character of $\pi_{\infty}$.

The integer $N$ appearing in the functional equation equals $\prod_{p} p^{a\left(\pi_{p}\right)}$, where $a\left(\pi_{p}\right)$ is the exponent of the conductor of the local representation $\pi_{p}$. Let $M=$ $\prod_{p} p^{a\left(\omega_{\pi, p}\right)}$, where $a\left(\omega_{\pi, p}\right)$ is the exponent of the conductor of $\omega_{\pi, p}$, the central character of $\pi_{p}$. By reduction to the supercuspidal case and using the existence of the distinguished vector exhibited in [12, one can easily prove that $a\left(\omega_{\pi, p}\right) \leq a\left(\pi_{p}\right)$. Consequently, $M \mid N$. We may therefore consider $\chi$, which originally was a Dirichlet 
character $\bmod M$, as a Dirichlet character $\bmod N$. This character is the character required by Axiom 5 .

By definition, $L(s, \pi)$ is an Euler product

$$
L(s, \pi)=\prod_{p \text { prime }} F_{p}\left(p^{-s}\right)^{-1},
$$

where each $F_{p}$ is a polynomial of degree at most $d$ (as required by Axiom 3(b)), with $F_{p}(0)=1$. Considering Langlands parameters at the nonarchimedean places, it follows from (2.5) that

$$
F_{p}(z)=1+\cdots+(-1)^{d} \chi(p) z^{d}
$$

as required by Axioms 3(a) and 5(a).

Considering Langlands parameters at the archimedean place, it follows that

$$
\omega_{\pi}(x)=x^{\operatorname{Im}\left(\sum \mu_{j}+\sum\left(2 \nu_{k}+1\right)\right)} \quad \text { for } x \in \mathbb{R}_{>0},
$$

and so, from (2.6), it follows that

$$
\chi(-1)=(-1)^{\sum \mu_{j}+\sum\left(2 \nu_{j}+1\right)},
$$

showing that $\mathbb{Q}$-automorphic $L$-functions satisfy Axioms 5(b) and 5(c).

Conjecturally, each local component $\pi_{p}$ of a cuspidal, automorphic representation $\pi$ as in Definition 2.3 is tempered; see [22] or [5. Conjecture 1.6]. The following lemma lists some consequences of temperedness for the spectral parameters and Satake parameters.

Lemma 3.2. Assume that $\pi=\bigotimes \pi_{p}$ is a cuspidal automorphic representation of $\mathrm{GL}\left(d, \mathbb{A}_{\mathbb{Q}}\right)$. Let $\mu_{j}, \nu_{j}$ be as in Theorem 3.1. Let the polynomial $F_{p}(z)$ in (3.3) be factored as

$$
F_{p}(z)=\left(1-\alpha_{1, p} z\right) \cdots\left(1-\alpha_{d_{p}, p} z\right)
$$

with $0 \leq d_{p} \leq d$ and $\alpha_{j, p} \in \mathbb{C}$.

(1) Assume that $\pi_{\infty}$ is tempered. Then for every $j$ we have $\operatorname{Re}\left(\mu_{j}\right) \in\{0,1\}$ and $\operatorname{Re}\left(\nu_{j}\right) \in\left\{\frac{1}{2}, 1, \frac{3}{2}, 2, \ldots\right\}$.

(2) Assume that $\pi_{p}$ is tempered for $p<\infty$ with $p \nmid N$. Then $\left|\alpha_{j, p}\right|=1$ for all $j \in\{1, \ldots, d\}$.

(3) Assume that $\pi_{p}$ is tempered for $p<\infty$ with $p \mid N$. Then $\left|\alpha_{j, p}\right|=p^{-m_{j} / 2}$ for some $m_{j} \in\{0,1,2, \ldots\}$, and $\sum m_{j} \leq d-d_{p}$.

This lemma implies that $\mathbb{Q}$-automorphic $L$-functions satisfy Axiom 4: the first item in the statement is about Axiom 4(a) and the second two are about Axiom 4(b).

Proof. (1) follows from the fact that a representation of $\mathrm{GL}(d, \mathbb{R})$ is tempered if and only if its Langlands parameter has bounded image. For (2), see 22] (also, (2) is a special case of (3)). For (3), we consider the local parameter of $\pi_{p}$, which is an admissible homomorphism $\varphi: W^{\prime}\left(\overline{\mathbb{Q}}_{p} / \mathbb{Q}_{p}\right) \rightarrow \operatorname{GL}(d, \mathbb{C})$. Here, $W^{\prime}\left(\overline{\mathbb{Q}}_{p} / \mathbb{Q}_{p}\right)$ is the Weil-Deligne group; see [20] for precise definitions and [14] for properties of the local Langlands correspondence. Let $\operatorname{sp}(n)$ be the $n$-dimensional indecomposable representation of $W^{\prime}\left(\overline{\mathbb{Q}}_{p} / \mathbb{Q}_{p}\right)$ defined in $[20, \S 5]$. We can write

$$
\varphi=\bigoplus_{j=1}^{t} \rho_{j} \otimes \operatorname{sp}\left(n_{j}\right),
$$


with uniquely determined irreducible representations $\rho_{j}$ of the Weil group $W\left(\overline{\mathbb{Q}}_{p} / \mathbb{Q}_{p}\right)$ and with uniquely determined positive integers $n_{i}$. Evidently, $d=$ $\sum_{j=1}^{t} \operatorname{dim}\left(\rho_{j}\right) n_{j}$. The Euler factor $L\left(s, \pi_{p}\right)$ is equal to the $L$-factor of $\varphi$, as defined in [20, §8]. By the Proposition in [20, $\S 8]$,

$$
L(s, \pi)=\prod_{j=1}^{t} L\left(s+n_{j}-1, \rho_{j}\right) .
$$

Assume that $\rho_{1}, \ldots, \rho_{r}$ are unramified characters of $W\left(\overline{\mathbb{Q}}_{p} / \mathbb{Q}_{p}\right)$ and that $\rho_{r+1}, \ldots, \rho_{t}$ are either ramified characters or of dimension greater than 1 . Then

$$
L(s, \pi)=\prod_{j=1}^{r} L\left(s+n_{j}-1, \rho_{j}\right)=\prod_{j=1}^{r} \frac{1}{1-\rho_{j}(p) p^{-s-n_{j}+1}},
$$

where we identified $\rho_{1}, \ldots, \rho_{r}$ with characters of $\mathbb{Q}_{p}^{\times}$. Comparison with (3.6) shows that $d_{p}=r$ and, after an appropriate permutation,

$$
\alpha_{j, p}=\rho_{j}(p) p^{-n_{j}+1} \quad \text { for } j=1, \ldots, r .
$$

Now $\pi_{p}$ is tempered if and only if the representations $|\cdot|_{p}^{\left(n_{i}-1\right) / 2} \otimes \rho_{i}$ are bounded for $i=1, \ldots, t$; see [14, §2.2]. In particular, assuming $\pi_{p}$ is tempered, we have $\left|\rho_{j}(p)\right|=p^{\left(n_{j}-1\right) / 2}$, and thus $\left|\alpha_{j, p}\right|=p^{-\left(n_{j}-1\right) / 2}$. Setting $m_{j}=n_{j}-1$, we have $\left|\alpha_{j, p}\right|=p^{-m_{j} / 2}$ and

$$
\sum_{j=1}^{d_{p}} m_{j}=\sum_{j=1}^{d_{p}} n_{j}-d_{p} \leq d-d_{p}
$$

This concludes the proof.

Proposition 3.3. Assume that $\pi=\bigotimes \pi_{p}$ is a cuspidal automorphic representation of $\mathrm{GL}\left(d, \mathbb{A}_{\mathbb{Q}}\right)$ such that each local component $\pi_{p}$ is tempered. Then $L(s, \pi)$ is a tempered analytic L-function in the sense of section 2.1. If $\pi$ is balanced, then $L(s, \pi)$ is balanced.

Proof. In the balanced case, this follows from Theorem 3.1, equations (3.3) and (3.5), and Lemma 3.2. Assume $\pi$ is not balanced. Then there exists $y \in \mathbb{R}$ such that $|\cdot|^{i y} \otimes \pi$ is balanced. Hence $L\left(s,|\cdot|{ }^{i y} \otimes \pi\right)=L(s+i y, \pi)$ is a balanced tempered analytic $L$-function. Consequently, $L(s, \pi)$ is a tempered analytic $L$-function.

\section{Algebraic And arithmetic $L$-Functions}

The most widely studied $L$-functions are those arising from arithmetic objects such as elliptic and higher-genus curves, holomorphic modular forms, number fields, Artin representations, Galois representations, and motives. We give two characterizations of such $L$-functions: one in terms of their Dirichlet coefficients and the other in terms of their spectral parameters.

4.1. Analytic $L$-functions of algebraic type. In [3] Booker, Strömbergsson, and Venkatesh - building on ideas of Stark and Hejhal - carried out computations that support the conjecture that if $\lambda>\frac{1}{4}$ is the Laplacian eigenvalue of a Maass form, then $\lambda$ is transcendental. Since the $\Gamma$-shifts in the associated $L$-function have imaginary part $\pm \sqrt{\lambda-\frac{1}{4}}$, one expects that the imaginary part of any $\Gamma$-shift in a 
primitive balanced analytic $L$-function is either 0 or transcendental. This motivates the following definition.

Definition 4.1. Suppose $L(s)$ is an analytic $L$-function with spectral parameters $\left\{\mu_{j}\right\}$ and $\left\{\nu_{k}\right\}$. We say that $L(s)$ is of algebraic type if either every $\mu_{j}$ and $\nu_{k}$ is in $\mathbb{Z}$ or every $\mu_{j}$ and $\nu_{k}$ is in $\frac{1}{2}+\mathbb{Z}$. The integer $w_{\text {alg }}=2 \max \left\{0, \nu_{1}, \ldots, \nu_{d_{2}}\right\}$ is called the algebraic weight of the $L$-function.

The second option, that $\mu_{j}$ and $\nu_{k}$ are in $\frac{1}{2}+\mathbb{Z}$, implies that there are no $\mu_{j}$, because if $L$ is tempered, then $\mu_{j} \in\{0,1\}$ and, in general (see Appendix $\mathrm{A}$ on the unitary pairing condition), $\mu_{j} \in\left(-\frac{1}{2}, \frac{1}{2}\right) \cup\left(\frac{1}{2}, \frac{3}{2}\right)$.

In [5. Definition 1.6], Clozel defined the notion of algebraic automorphic representation of GL $(n)$ over a number field. We use the term of algebraic type because our notion applies to the $L$-function, not its underlying representation; see section 4.3 for more details.

The term algebraic weight was chosen because if $M$ is a motive of weight $w$, then by Serre's recipe 26] $L(s, M)$ will have algebraic weight $w$.

4.2. Analytic $L$-functions of arithmetic type. The computations of Booker, Strömbergsson, and Venkatesh in [3] also support the conjecture that in general the Fourier coefficients of Maass forms with Laplacian eigenvalue $\lambda>\frac{1}{4}$ are transcendental and algebraically independent except for the constraints imposed by the Hecke relations. Thus we have a complement to the previous definition, involving a condition on the Dirichlet coefficients.

Definition 4.2. Suppose $L(s)=\sum a_{n} n^{-s}$ is an analytic $L$-function. We say that $L(s)$ is of arithmetic type if there exists $w_{\text {ar }} \in \mathbb{Z}$ and a number field $F$ such that $a_{n} n^{w_{\mathrm{ar}} / 2} \in \mathcal{O}_{F}$ for all $n$. The smallest such $F$ is called the field of coefficients, and the smallest such $w_{\text {ar }}$ is called the arithmetic weight of the $L$-function.

An analytic $L$-function with algebraic coefficients is not necessarily of arithmetic type, as shown by the example $L(s)=L(s, \chi) L(s, E)$, where $\chi$ is a primitive Dirichlet character and $E / \mathbb{Q}$ is an elliptic curve. As indicated in Figure2, it is conjectured that such examples must be nonprimitive.

As we will explain in Section 5, by combining existing conjectures, one obtains the conjecture that a primitive balanced analytic $L$-function is of algebraic type if and only if it is of arithmetic type. Furthermore, we have the Hodge conjecture: $w_{\mathrm{alg}}=w_{\mathrm{ar}}$.

4.3. $\mathbb{Q}$-automorphic $L$-functions of algebraic type. Let $\mathbb{A}_{F}$ be the ring of adeles of a number field $F$. In 5 , Clozel considered isobaric automorphic representations of $\operatorname{GL}\left(n, \mathbb{A}_{F}\right)$. He called such a representation algebraic if the local Langlands parameters at all archimedean places satisfy certain integrality conditions. More generally, for a connected, reductive $F$-group $G$ and an automorphic representation of $G\left(\mathbb{A}_{F}\right)$, Buzzard and Gee in [4] defined the notions of $C$-algebraic and $L$-algebraic. If $G=\mathrm{GL}(n)$ and $\pi$ is isobaric, then

$\pi$ is algebraic $\Longleftrightarrow \pi$ is $C$-algebraic $\Longleftrightarrow \pi|\cdot|^{\frac{n-1}{2}}$ is $L$-algebraic.

In the case of a tempered automorphic representation $\pi \cong \bigotimes \pi_{p}$ of $\operatorname{GL}\left(d, \mathbb{A}_{\mathbb{Q}}\right)$, the notions of $C$-algebraic and $L$-algebraic can easily be expressed in terms of the 
archimedean Euler factor. Recall that this factor is of the general form

$$
L\left(s, \pi_{\infty}\right)=\prod_{j=1}^{d_{1}} \Gamma_{\mathbb{R}}\left(s+\mu_{j}\right) \prod_{k=1}^{d_{2}} \Gamma_{\mathbb{C}}\left(s+\nu_{k}\right)
$$

with complex numbers $\mu_{j}$ and $\nu_{k}$, and $d=d_{1}+2 d_{2}$.

Lemma 4.3. Let $\pi \cong \bigotimes \pi_{p}$ be an automorphic representation of $\mathrm{GL}\left(d, \mathbb{A}_{\mathbb{Q}}\right)$ such that $\pi_{\infty}$ is tempered. Let $L\left(s, \pi_{\infty}\right)$ be as in (4.1).

(1) Assume that $d$ is even. Then

- $\pi$ is C-algebraic if and only if $d_{1}=0$ and $\nu_{k} \in \frac{1}{2}+\mathbb{Z}_{\geq 0}$ for $k=$ $1, \ldots, d / 2$.

- $\pi$ is L-algebraic if and only if $\mu_{j} \in\{0,1\}$ for $j=1, \ldots, d_{1}$ and $\nu_{k} \in$ $\mathbb{Z}_{>0}$ for $k=1, \ldots, d_{2}$.

(2) Assume that $d$ is odd. Then $\pi$ is $C$-algebraic if and only if $\pi$ is $L$-algebraic if and only if $\mu_{j} \in\{0,1\}$ for $j=1, \ldots, d_{1}$ and $\nu_{k} \in \mathbb{Z}_{>0}$ for $k=1, \ldots, d_{2}$.

Proof. This follows in a straightforward manner from 4, Definitions 5.7 and 5.9] and the well known recipe of attaching $\Gamma$-factors to representations of the real Weil group.

Remark 4.4. Suppose $L(s, \pi)$ is a $\mathbb{Q}$-automorphic $L$-function, coming from a unitary cuspidal automorphic representation $\pi=\bigotimes_{p \leq \infty} \pi_{p}$ of $\mathrm{GL}\left(d, \mathbb{A}_{\mathbb{Q}}\right)$, as in Definition 2.3. In particular, as shown in the course of Section 3, $L(s, \pi)$ is an analytic $L$-function. Thus, we can say that $L(s, \pi)$ is of algebraic type if it satisfies the conditions of Definition 4.1

Therefore, by the lemma we have:

Remark 4.5. By the Ramanujan conjecture, all local components $\pi_{p}$ of the cuspidal automorphic representation $\pi$ are tempered. Assuming this is the case, we see that $L(s, \pi)$ is of algebraic type if and only if $\pi$ is either $C$-algebraic or $L$-algebraic.

4.4. $\mathbb{Q}$-automorphic $L$-functions of arithmetic type. Let $G$ be a connected, reductive group over the number field $F$, and let $\pi \cong \bigotimes \pi_{v}$ be an automorphic representation of $G\left(\mathbb{A}_{F}\right)$. Buzzard and Gee [4] define the notions of $\pi$ being $C$ arithmetic and L-arithmetic in terms of the Satake parameters of $\pi_{v}$ at almost all places. For $G=\mathrm{GL}(n)$ it is true that

$$
\pi \text { is } C \text {-arithmetic } \Longleftrightarrow \pi|\cdot|^{\frac{n-1}{2}} \text { is } L \text {-arithmetic. }
$$

The conditions can easily be reformulated in terms of $L$-functions:

Lemma 4.6. Let $\pi \cong \bigotimes \pi_{p}$ be an automorphic representation of $\mathrm{GL}\left(d, \mathbb{A}_{\mathbb{Q}}\right)$. Let $S$ be a finite set of primes such that $\pi_{p}$ is unramified for primes $p \notin S$. Let

$$
L\left(s, \pi_{p}\right)=\frac{1}{\left(1-\alpha_{p, 1} p^{-s}\right) \cdot \ldots \cdot\left(1-\alpha_{p, d} p^{-s}\right)}
$$

be the Euler factor for $p \notin S$.

(1) Assume that $d$ is even. Then

- $\pi$ is $C$-arithmetic if and only if there exists a number field $E$ such that $\alpha_{p, 1} \sqrt{p}, \ldots, \alpha_{p, d} \sqrt{p} \in E$ for almost all $p \notin S$.

- $\pi$ is L-arithmetic if and only if there exists a number field $E$ such that $\alpha_{p, 1}, \ldots, \alpha_{p, d} \in E$ for almost all $p \notin S$. 
(2) Assume that $d$ is odd. Then $\pi$ is C-arithmetic if and only if $\pi$ is $L$ arithmetic if and only if there exists a number field $E$ such that $\alpha_{p, 1}, \ldots$, $\alpha_{p, d} \in E$ for almost all $p \notin S$.

The following is equivalent to Definition 4.2. but we include this formulation because it is stated in terms of the parameters which are more natural for $\mathbb{Q}$ automorphic $L$-functions.

Definition 4.7. Let $L(s, \pi)$ be a $\mathbb{Q}$-automorphic $L$-function, coming from a cuspidal automorphic representation $\pi=\bigotimes_{p \leq \infty} \pi_{p}$ of $\mathrm{GL}\left(d, \mathbb{A}_{\mathbb{Q}}\right)$, as in Definition 2.3. Let

$$
L\left(s, \pi_{p}\right)=\frac{1}{\left(1-\alpha_{p, 1} p^{-s}\right) \cdot \ldots \cdot\left(1-\alpha_{p, d} p^{-s}\right)}
$$

be the Euler factor at a prime $p$. We say that $L(s, \pi)$ is of arithmetic type if there exists a number field $E$ such that either

$$
\alpha_{p, 1}, \ldots, \alpha_{p, d} \in E \quad \text { for almost all good primes } p
$$

or

$$
\alpha_{p, 1} \sqrt{p}, \ldots, \alpha_{p, d} \sqrt{p} \in E \quad \text { for almost all good primes } p .
$$

Remark 4.8. Let $\pi$ be as in Definition 4.7, and suppose $d$ is odd. Then (4.5) cannot occur. Indeed, if (4.5) would hold, then we would also have

$$
\alpha_{p, 1} \cdot \ldots \cdot \alpha_{p, d} \sqrt{p} \in E \quad \text { for almost all good primes } p \text {. }
$$

But the numbers $\alpha_{p, 1} \cdot \ldots \cdot \alpha_{p, d}$ are the Satake parameters of the central character $\omega_{\pi}$ of $\pi$, which, up to a unitary twist, corresponds to a Dirichlet character $\chi$; see (2.5). Hence we would have

$$
\chi(p) p^{i t} \sqrt{p}=\chi(p) p^{i t+\frac{1}{2}} \in E \quad \text { for almost all good primes } p
$$

for some real number $t$, so in particular

$$
p^{i t+\frac{1}{2}} \in E \quad \text { for infinitely many } p .
$$

This is impossible for $t \in \mathbb{R}$ because of the following consequence of the Six Exponentials Theorem [30].

Lemma 4.9. If $\alpha \in \mathbb{C}$ and $E / \mathbb{Q}$ is a number field with $p^{\alpha} \in E$ for infinitely many primes $p$, then $\alpha \in \mathbb{Z}$.

Proof. Suppose $\alpha \notin \mathbb{Z}$. We cannot have $\alpha \in \mathbb{Q}$ because $E$ is a finite extension of $\mathbb{Q}$. Thus, $\{1, \alpha\}$ is linearly independent over the rationals. (We also cannot have $\alpha$ algebraic because, by the Gelfond-Schneider theorem, $p^{\alpha}$ would be transcendental. This does not seem to be needed in the proof.)

Suppose $p_{1}, p_{2}$, and $p_{3}$ are distinct primes with $p_{j}^{\alpha} \in E$. Since $\left\{\log p_{1}, \log p_{2}\right.$, $\left.\log p_{3}\right\}$ is linearly independent over the rationals, we have a contradiction because by the Six Exponentials Theorem [30, one of $p_{1}, p_{2}, p_{3}, p_{1}^{\alpha}, p_{2}^{\alpha}$, and $p_{3}^{\alpha}$ must be transcendental.

As a consequence of Lemma 4.6 and the above remark, we see that $L(s, \pi)$ is of arithmetic type if and only if $\pi$ is either $C$-arithmetic or $L$-arithmetic (see Section 5]. So the conjectures of Clozel [5] and Buzzard and Gee 4] yield: 
Conjecture 4.10. A $\mathbb{Q}$-automorphic L-function is of algebraic type if and only if it is of arithmetic type.

A more general conjecture for automorphic representations $\pi$ of $G\left(\mathbb{A}_{F}\right)$ was made by Buzzard and Gee in [4. Namely, $\pi$ is $L$-algebraic if and only if it is $L$-arithmetic, and $\pi$ is $C$-algebraic if and only if it is $C$-arithmetic. The surprising fact about these conjectures is that a condition purely in terms of archimedean $L$-parameters is conjecturally equivalent to a condition purely in terms of nonarchimedean $L$ parameters.

For isobaric automorphic representations of $\operatorname{GL}\left(n, \mathbb{A}_{F}\right)$, the conjecture that $C$ algebraic implies $C$-arithmetic is also a consequence of the more general conjectures made in [5,6]; see [4, $\S 8.1]$.

4.5. $L$-functions of motives. Let $F$ be a number field. The category of motives over $F$ was constructed by Grothendieck in the 1960s. The first article about motives seems to be [9]. More contemporary and useful surveys are [13, [23], and [17]. We will not recall here the construction of the category of motives. What is important for us is that, given a pure motive $M$ of weight $w$, there is an $L$ function $L(M, s)$ attached to it, which, after completing it to a function $\Lambda(M, s)$ using appropriate $\Gamma$-factors, conjecturally satisfies a functional equation $\Lambda(M, s)=$ $\pm \bar{\Lambda}(M, 1+w-s)$. We write $\Lambda(M, s)$ and not $\Lambda(s, M)$, because the functional equation relates $s$ and $1+w-s$, and not $s$ and $1-s$.

We briefly recall the shape of $\Lambda(M, s)$, using [26] as our reference. We assume that the ground field is $\mathbb{Q}$ for simplicity. For each nonarchimedean place $p$ the characteristic polynomial $P_{w, p}$ of the action of Frobenius on the inertia-fixed points of the $w$ th étale cohomology group (at least conjecturally) has coefficients in $\mathbb{Z}$. We set

$$
L_{p}(M, s)=\frac{1}{P_{w, p}\left(p^{-s}\right)},
$$

and $L(M, s)=\prod_{p<\infty} L_{p}(M, s)$. There exists a positive integer $d$, called the rank of $M$, such that for almost all places the polynomial $P_{w, p}$ will have degree $d$. For all "good" places $p$, if we factor

$$
P_{w, p}(T)=\prod_{j=1}^{b_{w}}\left(1-\alpha_{j, p} T\right), \quad \alpha_{j, p} \in \mathbb{C}^{\times},
$$

then it is conjectured that

$$
\left|\alpha_{j, p}\right|=p^{w / 2}
$$

(see $C_{7}$ in $\S 2.3$ of [26]). At the archimedean place $\infty$ we have

$$
L_{\infty}(M, s)=\Gamma_{\mathbb{R}}\left(s-\frac{w}{2}\right)^{h^{w / 2,+}} \Gamma_{\mathbb{R}}\left(s-\frac{w}{2}+1\right)^{h^{w / 2,-}} \prod_{\substack{p+q=w \\ p<q}} \Gamma_{\mathbb{C}}(s-p)^{h^{p, q}},
$$

with the first two factors only appearing if $w$ is even. Here, the $h^{p, q}$ are the dimensions of the spaces $H^{p, q}$ in the Hodge decomposition of the Betti realization of $M$. If $w$ is even, then there is a space $H^{p, p}(p=w / 2)$, on which complex conjugation acts as an involution; the number $h^{p, \pm}$ is the dimension of the \pm 1 -eigenspace.

Conjecturally, there exists a positive integer $N$ such that

$$
\Lambda(M, s)=N^{(s-w / 2) / 2} L_{\infty}(M, s) L(M, s)
$$


extends to an entire function on all of $\mathbb{C}$, is bounded in vertical strips, and satisfies the functional equation $\Lambda(M, s)= \pm \bar{\Lambda}(M, 1+w-s)$.

Replacing $s$ by $s+w / 2$, we obtain the analytically normalized functions $L(s, M:=$ $L(M, s+w / 2)$ and $\Lambda(s, M):=\Lambda(M, s+w / 2)$. The functional equation becomes $\Lambda(s, M)= \pm \bar{\Lambda}(1-s, M)$. The factor (4.12) turns into

$$
L_{\infty}(M, s+w / 2)=\Gamma_{\mathbb{R}}(s)^{h^{w / 2,+}} \Gamma_{\mathbb{R}}(s+1)^{h^{w / 2,-}} \prod_{\substack{p+q=w \\ p<q}} \Gamma_{\mathbb{C}}\left(s+\frac{q-p}{2}\right)^{h^{p, q}}
$$

By (4.11), the roots of the denominator polynomials of $L(s, M)$ will have absolute value 1 .

As described in [5, §4.3]:

Conjecture 4.11. There exists a one-to-one correspondence between irreducible, pure motives $M$ over $\mathbb{Q}$ of rank $d$, and $C$-algebraic cuspidal automorphic representations $\pi$ of $\mathrm{GL}\left(d, \mathbb{A}_{\mathbb{Q}}\right)$, such that $L(s, M)=L(s, \pi)$.

The conjecture implies that the class of analytically normalized $L$-functions arising from irreducible, pure motives is the same as the class of $\mathbb{Q}$-automorphic $L$ functions of algebraic type. The conjecture that $L(s, M)$ should satisfy the required analytic properties shared by the class of analytic $L$-functions as defined in Section 2.1 is known as the Hasse-Weil conjecture.

Remark 4.12. Given a $\Gamma$-factor in the analytic normalization (4.14), it is not possible to determine the weight of the underlying motive. Indeed, the motives which (conjecturally) are attached to the $L$-function form an equivalence class, where the members are Tate twists of each other. It is natural to choose a twist so that nonvanishing Hodge numbers of that motive are among $\left(h^{w, 0}, \ldots, h^{0, w}\right)$, with $h^{w, 0}=h^{0, w}>0$. The weight, $w$, of that motive will equal the algebraic weight of the $L$-function, which explains our choice of terminology.

4.6. $L$-functions of Galois representations. Let $\operatorname{Gal}(\overline{\mathbb{Q}} / \mathbb{Q})$ be the absolute Galois group of $\mathbb{Q}$. Let $L$ be a finite extension of $\mathbb{Q}_{\ell}$ for some prime $\ell$. A continuous homomorphism $\rho: \operatorname{Gal}(\overline{\mathbb{Q}} / \mathbb{Q}) \rightarrow \operatorname{GL}(d, L)$ will be referred to as a Galois representation; see 25] for basic facts. In [10] the class of geometric Galois representations was defined. As summarized in Taylor [29, Galois representations arising from motives are geometric. Conversely, the Fontaine-Mazur conjecture asserts that any geometric Galois representation is motivic. Hence, the class of (analytically normalized) $L$-functions arising from geometric Galois representations should be the same as the class of (analytically normalized) $L$-functions attached to Galois representations. Assuming Conjecture 4.11 this is also the same as the class of $\mathbb{Q}$ automorphic $L$-functions of algebraic type. This explains the triangle in the upper left corner of Figure 2 ,

Conjecture 5.16 of $[4$, for $G=\mathrm{GL}(d)$ over $\mathbb{Q}$, makes the statement that a geometric Galois representation is attached to an $L$-algebraic automorphic representation $\pi$ of $\operatorname{GL}\left(d, \mathbb{A}_{\mathbb{Q}}\right)$; for recent progress on this conjecture, see [28] and [11. That every Galois representation arises from an automorphic representation is known as the Modularity Conjecture. 


\section{Connections}

The equalities between sets of $L$-functions in Figure 1 are a consequence of the 12 relations shown in Figure 2, where an arrow means "can be viewed as a natural subset of". Most of those arrows are at least partially conjectural. More detailed explanations of these arrows can be found in Tables 1 and 2 .

TABLE 1. Explanations of the arrows in Figure2 between analytic $L$-functions and $\mathbb{Q}$-automorphic $L$-functions. These explanations and these arrows correspond to Section 3 and Sections 4.14 .4 If the justification for a connection is "formal", this means that it is an immediate corollary of the axioms or properties satisfied by the sets of $L$-functions being connected.

\begin{tabular}{|c|c|c|}
\hline Connection & Label & Justification \\
\hline$Q \subset A$ & J-PS-S & $\begin{array}{l}\text { A result generally due to Cogdell- } \\
\text { Piatetski-Shapiro and Jacquet-Piatetski- } \\
\text { Shapiro-Shalika [7, 12] as formulated in } \\
\text { Proposition 3.3. }\end{array}$ \\
\hline$A \subset Q$ & $\mathrm{~S}$ & $\begin{array}{l}\text { Selberg } 24 \text { identified a class of axioms } \\
\text { for } L \text {-functions and implicitly conjectured } \\
\text { that this class is contained in the class as- } \\
\text { sociated to automorphic representations } \\
\text { as defined by Langlands [15]. } A \text { and } Q \\
\text { are, respectively, subsets of these classes } \\
\text { with the same formal properties. }\end{array}$ \\
\hline$Q_{*} \subset Q$ & & Restriction to a subset. \\
\hline$Q_{1} \cup Q_{2}=Q_{*}$ & & Remark 4.5 . \\
\hline$Q_{1}=Q_{2}$ & B-G & $\begin{array}{l}\text { A conjecture due to Buzzard-Gee [4] as } \\
\text { formulated in Conjecture } 4.10 \text {, }\end{array}$ \\
\hline$A_{*} \subset A$ & & Restriction to a subset. \\
\hline$Q_{1}=A_{1}$ & & Formal, if we assume $A=Q$. \\
\hline$Q_{2} \subset A_{2}$ & & $\begin{array}{l}\text { A result of Clozel [5] implies that the } \\
\text { (suitably) rescaled coefficients are inte- } \\
\text { gers and the inclusion otherwise is formal. }\end{array}$ \\
\hline$A_{2} \subset Q_{2}$ & & Formal. \\
\hline$A_{1}=A_{2}$ & & Piecing together previous connections. \\
\hline $\begin{aligned} A_{*} & =A_{1}=A_{2} \\
& =Q_{1}=Q_{2}=Q_{*}\end{aligned}$ & & Piecing together previous connections. \\
\hline
\end{tabular}




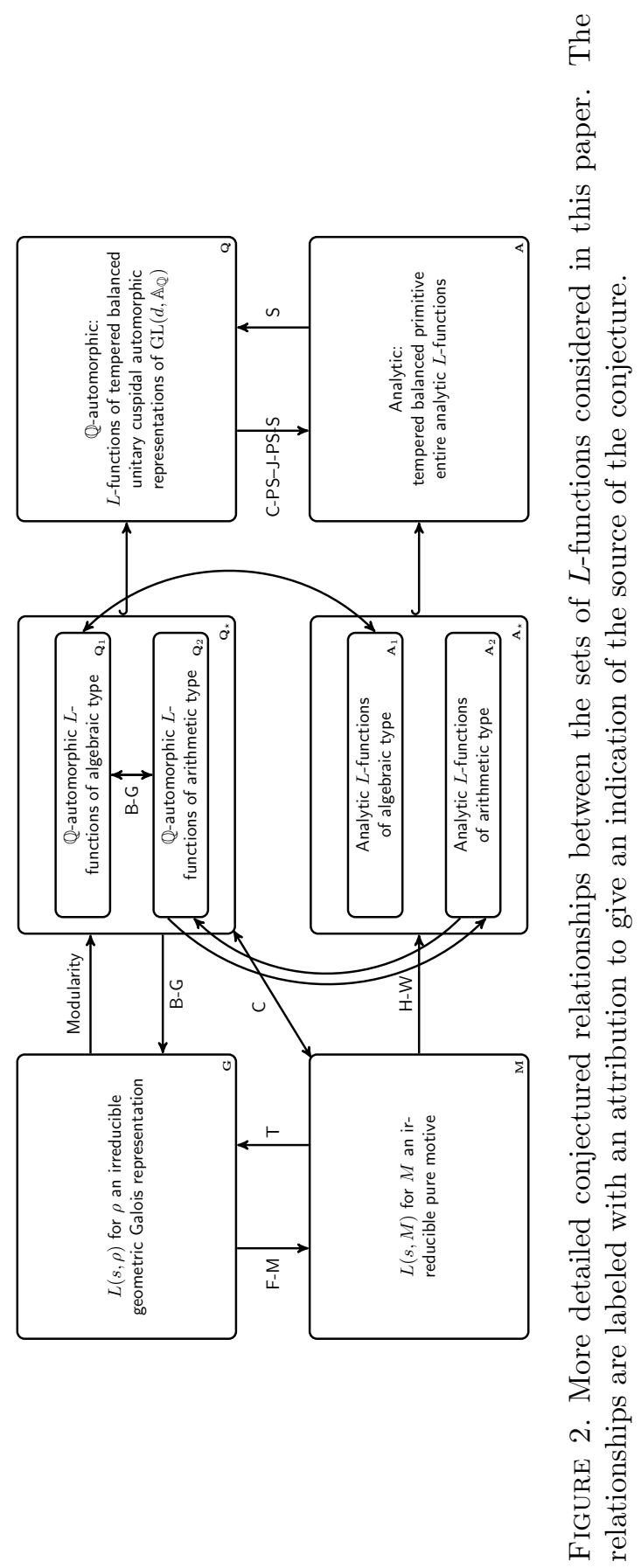


TABLE 2. Explanations of arrows in Figure 2 between various sources of $L$-functions as described in Sections 4.5 and 4.6 .

\begin{tabular}{|c|c|c|}
\hline Connection & Label & Justification \\
\hline$M \subset A_{*}$ & $\mathrm{H}-\mathrm{W}$ & $\begin{array}{l}\text { In our notation this is a restatement of } \\
\text { the Hasse-Weil conjecture. }\end{array}$ \\
\hline$M=Q_{*}$ & $\mathrm{C}$ & Conjecture due to Clozel $[5,6]$ \\
\hline$M \subset G$ & $\mathrm{~T}$ & $\begin{array}{l}\text { Taylor [29, pp, } 77,79-80] \text { distills the work } \\
\text { of many people and describes how to at- } \\
\text { tach a Galois representation to a motive. }\end{array}$ \\
\hline$G \subset M$ & F-M & Fontaine-Mazur conjecture [10]. \\
\hline$G \subset Q_{*}$ & Modularity & $\begin{array}{l}\text { The general conjecture asserts that to a } \\
\text { Galois representation one can attach an } \\
\text { automorphic representation so that the } \\
\text { two } L \text {-functions agree. }\end{array}$ \\
\hline$Q_{*} \subset G$ & B-G & Conjecture due to Buzzard-Gee [4]. \\
\hline$M=A_{*}=Q_{*}=G$ & & Piecing together previous connections. \\
\hline
\end{tabular}

\section{Appendix A. Nontempered $L$-Functions}

If an $L$-function fails to be tempered, the failure could either occur in the $\Gamma$ factors or in the local factors of the Euler product. The failure must occur in a specific form, which we call the unitary pairing condition. Our motivation is that the unitary pairing condition holds for the factors arising from generic unitary local representations.

A.1. The unitary pairing condition at infinity. In the definition we use the following notation: if $x \in \mathbb{R}$ and $\xi \in \mathbb{C}$, then $(x, \xi)^{*}=(x,-\bar{\xi})$. Also, we introduce a parameter $\theta<\frac{1}{2}$ which measures how far the $L$-function is from being tempered at infinity.

Definition A.1. The multisets $\left\{\mu_{j}\right\}$ and $\left\{\nu_{j}\right\}$ meet the unitary pairing condition at infinity if it is possible to write $\mu_{j}=\delta_{j}+\alpha_{j}$ and $\nu_{j}=\eta_{j}+\beta_{j}$, where $\delta_{j} \in\{0,1\}$ and $\eta_{j} \in\left\{\frac{1}{2}, 1, \frac{3}{2}, \ldots\right\}$, with $\left|\operatorname{Re}\left(\alpha_{j}\right)\right|,\left|\operatorname{Re}\left(\beta_{j}\right)\right|<\theta$, such that the multisets $\left\{\left(\delta_{j}, \alpha_{j}\right)\right\}$ and $\left\{\left(\eta_{j}, \beta_{j}\right)\right\}$ are closed under the operation $S \rightarrow S^{*}$.

For example, the following $\Gamma$-factor satisfies the unitary pairing condition:

$$
\begin{aligned}
\Gamma_{\mathbb{R}}(s- & 0.2) \Gamma_{\mathbb{R}}(s+0.2) \Gamma_{\mathbb{R}}(s)^{3} \Gamma_{\mathbb{R}}(s+0.9) \Gamma_{\mathbb{R}}(s+1.1) \\
& \times \Gamma_{\mathbb{C}}(s+0.7) \Gamma_{\mathbb{C}}(s+1.3)^{2} \Gamma_{\mathbb{C}}(s+1.7) \Gamma_{\mathbb{C}}(s+7),
\end{aligned}
$$

as does this one

$$
\begin{aligned}
\Gamma_{\mathbb{R}}(s- & 0.2+3 i) \Gamma_{\mathbb{R}}(s+0.2+3 i) \Gamma_{\mathbb{R}}(s+1) \Gamma_{\mathbb{R}}(s+1-8 i) \\
& \times \Gamma_{\mathbb{C}}(s+0.7) \Gamma_{\mathbb{C}}(s+1.3) \Gamma_{\mathbb{C}}(s+1.3-7 i) \Gamma_{\mathbb{C}}(s+1.7-7 i) .
\end{aligned}
$$

A.2. The unitary pairing condition at $p$. Just as in the archimedean case, we introduce a parameter $\theta<\frac{1}{2}$ which provides a weak version of the Ramanujan bound: $\left|\alpha_{j, p}\right| \leq p^{\theta}$. At a good prime the unitary pairing condition is easy to state. 
Definition A.2. Suppose $p$ is a good prime. The multiset $\left\{\alpha_{1}, \ldots, \alpha_{d}\right\}$ meets the unitary pairing condition at $p$ with partial Ramanujan bound $\theta<\frac{1}{2}$, if $\left|\alpha_{j}\right| \leq p^{\theta}$ and the multiset is closed under the operation $x \rightarrow 1 / \bar{x}$. Equivalently, the polynomial $F(z)=\prod_{j}\left(1-\alpha_{j} z\right)$ has all its roots in $|z| \geq p^{-\theta}$ and satisfies the self-reciprocal condition

$$
F(z)=\xi z^{d} \bar{F}\left(z^{-1}\right)
$$

where $\xi=(-1)^{d} \prod_{j} \alpha_{j}$.

The term self-reciprocal refers to the fact that, up to multiplication by a constant, the coefficients of the polynomial are the same if read in either order.

If $\left|\alpha_{j}\right|=1$, then the unitary pairing condition at $p$ says nothing, because $\alpha_{j}=$ $1 / \overline{\alpha_{j}}$. But those Satake parameters which are not on the unit circle occur in pairs: if $\alpha_{j}=r e^{i \theta}$ with $r \neq 1$, then $r^{-1} e^{i \theta}$ is also a Satake parameter. Those two points are located symmetrically with respect to the unit circle.

The general case of the unitary pairing condition at $p$, which includes the good prime version above, closely follows the archimedean case. Specifically, the $\Gamma_{\mathbb{R}}$ factors are like the good primes, and the $\Gamma_{\mathbb{C}}$ factors are similar to the bad primes.

Recall the notation $(x, \xi)^{*}=(x,-\bar{\xi})$.

Definition A.3. The multiset $\left\{\alpha_{1}, \ldots, \alpha_{M}\right\}$ meets the unitary pairing condition at $p$ of degree $d$ and partial Ramanujan bound $\theta<\frac{1}{2}$, if it is possible to write $\alpha_{j}=p^{-\eta_{j}-\beta_{j}}$ where $\eta_{j} \in\left\{0, \frac{1}{2}, 1, \frac{3}{2}, \ldots\right\}$, with $\sum 2 \eta_{j} \leq d-M$ and $\left|\operatorname{Re}\left(\beta_{j}\right)\right| \leq \theta$, such that the multiset $S=\left\{\left(\eta_{j}, \beta_{j}\right)\right\}$ is closed under the operation $S \rightarrow S^{*}$.

\section{About THE AUthors}

David Farmer works at the American Institute of Mathematics in San Jose, California. In addition to doing research on $L$-functions, he helps run AIM's workshop program, builds tools to support research mathematics, and is part of a team that is creating the next generation of free online textbooks.

Ameya Pitale is associate professor at the University or Oklahoma. He received his $\mathrm{PhD}$ in 2006. His main research interest is in the Langlands program. He works on research problems in classical modular forms and automorphic representation theory.

Nathan Ryan is associate professor of mathematics at Bucknell University. He received his $\mathrm{PhD}$ in 2005. His main research interests are in the computational aspects of $L$-functions and modular forms. He is particularly interested in Siegel modular forms and their $L$-functions.

Ralf Schmidt is professor of mathematics at the University of Oklahoma. He received his $\mathrm{PhD}$ in 1998. His main research interests are in classical and adelic automorphic forms, particularly Siegel modular forms, and the associated local and global representation theory.

\section{REFERENCES}

[1] J. Arthur, The endoscopic classification of representations, American Mathematical Society Colloquium Publications, vol. 61, American Mathematical Society, Providence, RI, 2013. Orthogonal and symplectic groups. MR 3135650

[2] J. Arthur and L. Clozel, Simple algebras, base change, and the advanced theory of the trace formula, Annals of Mathematics Studies, vol. 120, Princeton University Press, Princeton, NJ, 1989. MR 1007299 
[3] A. R. Booker, A. Strömbergsson, and A. Venkatesh, Effective computation of Maass cusp forms, Int. Math. Res. Not., posted on 2006, Art. ID 71281, 34, DOI 10.1155/IMRN/2006/71281. MR2249995

[4] K. Buzzard and T. Gee, The conjectural connections between automorphic representations and Galois representations, Automorphic forms and Galois representations. Vol. 1, London Math. Soc. Lecture Note Ser., vol. 414, Cambridge Univ. Press, Cambridge, 2014, pp. 135187, DOI 10.1017/CBO9781107446335.006. MR3444225

[5] L. Clozel, Motifs et formes automorphes: applications du principe de fonctorialité (French), Automorphic forms, Shimura varieties, and $L$-functions, Vol. I (Ann Arbor, MI, 1988), Perspect. Math., vol. 10, Academic Press, Boston, MA, 1990, pp. 77-159. MR.1044819

[6] L. Clozel, Motives and automorphic representations, Autour des motifs-École d'été FrancoAsiatique de Géométrie Algébrique et de Théorie des Nombres/Asian-French Summer School on Algebraic Geometry and Number Theory. Vol. III, Panor. Synthèses, vol. 49, Soc. Math. France, Paris, 2016, pp. 29-60. MR 3642468

[7] J. W. Cogdell and I. I. Piatetski-Shapiro, Remarks on Rankin-Selberg convolutions, Contributions to automorphic forms, geometry, and number theory, Johns Hopkins Univ. Press, Baltimore, MD, 2004, pp. 255-278. MR2058610

[8] J. B. Conrey and A. Ghosh, On the Selberg class of Dirichlet series: small degrees, Duke Math. J. 72 (1993), no. 3, 673-693, DOI 10.1215/S0012-7094-93-07225-0. MR1253620

[9] Michel Demazure, Motifs des variétés algébriques, Séminaire Bourbaki Vol. 1969, Exp. 365.

[10] J.-M. Fontaine and B. Mazur, Geometric Galois representations, Elliptic curves, modular forms, and Fermat's last theorem (Hong Kong, 1993), Ser. Number Theory, I, Int. Press, Cambridge, MA, 1995, pp. 41-78. MR1363495

[11] M. Harris, K.-W. Lan, R. Taylor, and J. Thorne, On the rigid cohomology of certain Shimura varieties, Res. Math. Sci. 3 (2016), Paper No. 37, 308, DOI 10.1186/s40687-016-0078-5. MR.3565594

[12] H. Jacquet, I. I. Piatetski-Shapiro, and J. Shalika, Conducteur des représentations du groupe linéaire (French), Math. Ann. 256 (1981), no. 2, 199-214, DOI 10.1007/BF01450798. MR620708

[13] S. L. Kleiman, The standard conjectures, Motives (Seattle, WA, 1991), Proc. Sympos. Pure Math., vol. 55, Amer. Math. Soc., Providence, RI, 1994, pp. 3-20, DOI 10.1090/pspum/055.1/1265519. MR1265519

[14] S. S. Kudla, The local Langlands correspondence: the non-Archimedean case, Motives (Seattle, WA, 1991), Proc. Sympos. Pure Math., vol. 55, Amer. Math. Soc., Providence, RI, 1994, pp. 365-391. MR1265559

[15] R. P. Langlands, L-functions and automorphic representations, Proceedings of the International Congress of Mathematicians (Helsinki, 1978), Acad. Sci. Fennica, Helsinki, 1980, pp. 165-175. MR562605

[16] J. Liu and Y. Ye, Weighted Selberg orthogonality and uniqueness of factorization of automorphic L-functions, Forum Math. 17 (2005), no. 3, 493-512, DOI 10.1515/form.2005.17.3.493. MR2138503

[17] J. S. Milne, Motives - Grothendieck's dream, Open problems and surveys of contemporary mathematics, Surv. Mod. Math., vol. 6, Int. Press, Somerville, MA, 2013, pp. 325-342. MR.3204952

[18] A. Pitale, A. Saha, and R. Schmidt, Transfer of Siegel cusp forms of degree 2, Mem. Amer. Math. Soc. 232 (2014), no. 1090, vi+107. MR3243731

[19] A. Raghuram and N. Tanabe, Notes on the arithmetic of Hilbert modular forms, J. Ramanujan Math. Soc. 26 (2011), no. 3, 261-319. MR2865819

[20] D. E. Rohrlich, Elliptic curves and the Weil-Deligne group, Elliptic curves and related topics, CRM Proc. Lecture Notes, vol. 4, Amer. Math. Soc., Providence, RI, 1994, pp. 125-157. MR.1260960

[21] Z. Rudnick and P. Sarnak, Zeros of principal L-functions and random matrix theory, Duke Math. J. 81 (1996), no. 2, 269-322, DOI 10.1215/S0012-7094-96-08115-6. A celebration of John F. Nash, Jr. MR1395406

[22] P. Sarnak, Notes on the generalized Ramanujan conjectures, Harmonic analysis, the trace formula, and Shimura varieties, Clay Math. Proc., vol. 4, Amer. Math. Soc., Providence, RI, 2005, pp. 659-685. MR2192019 
[23] A. J. Scholl, Classical motives, Motives (Seattle, WA, 1991), Proc. Sympos. Pure Math., vol. 55, Amer. Math. Soc., Providence, RI, 1994, pp. 163-187, DOI 10.1090/pspum/055.1/1265529. MR1265529

[24] A. Selberg, Old and new conjectures and results about a class of Dirichlet series, Proceedings of the Amalfi Conference on Analytic Number Theory (Maiori, 1989), Univ. Salerno, Salerno, 1992, pp. 367-385. MR.1220477

[25] J.-P. Serre, Abelian l-adic representations and elliptic curves, McGill University lecture notes written with the collaboration of Willem Kuyk and John Labute, W. A. Benjamin, Inc., New York-Amsterdam, 1968. MR 0263823

[26] J. P. Serre, Facteurs locaux des fonctions zêta des variétés algébriques, Séminaire DelangePisot-Poitou, Théories des Nombres 11 (1969-1970), no. 2, 1-15.

[27] F. Shahidi, On certain L-functions, Amer. J. Math. 103 (1981), no. 2, 297-355, DOI 10.2307/2374219. MR610479

[28] S. W. Shin, Galois representations arising from some compact Shimura varieties, Ann. of Math. (2) 173 (2011), no. 3, 1645-1741, DOI 10.4007/annals.2011.173.3.9. MR2800722

[29] R. Taylor, Galois representations, Proceedings of the International Congress of Mathematicians, Vol. I (Beijing, 2002), Higher Ed. Press, Beijing, 2002, pp. 449-474. MR1989198

[30] M. Waldschmidt, On the transcendence methods of Gel'fond and Schneider in several variables, New advances in transcendence theory (Durham, 1986), Cambridge Univ. Press, Cambridge, 1988, pp. 375-398. MR972013

American Institute of Mathematics, 600 East Brokaw Road, San Jose, California 95112-1006

Email address: farmer@aimath.org

Department of Mathematics, University of Oklahoma, Norman, Oklahoma 73019-3103

Email address: apitale@math.ou.edu

Department of Mathematics, Bucknell University, Lewisburg, Pennsylvania 17837

Email address: nathan.ryan@bucknell.edu

Department of Mathematics, University of Oklahoma, Norman, Oklahoma 73019-3103

Email address: rschmidt@math.ou.edu 